\title{
Highlights from ABC4 - Bridging the Gap
}

\author{
Chair: $\quad$ Rachel Wuerstlein ${ }^{a}$ \\ Participants: Johannes Ettl ${ }^{b} \quad$ Renate Haidinger $^{c}$ Diana Lueftner ${ }^{d} \quad$ Joana Ribeiro $^{\text {e }}$ \\ Janice Tsang ${ }^{f}$ \\ ${ }^{a}$ Breast Center, Dept. Of Gynecology and Obstetrics and CCC Munich, University of Munich (LMU), Munich, Germany; \\ ${ }^{b}$ Frauenklinik, Klinikum rechts der Isar, Technical University Munich, Munich, Germany; \\ ${ }^{\mathrm{c}}$ Brustkrebs Deutschland e.V., Hohenbrunn, Germany; \\ d Medical Clinic focusing on Hematology, Oncology and Tumor Immunology, Charité Berlin, Benjamin Franklin Campus, Berlin, Germany; \\ e Champalimaud Clinical Centre, Lisbon, Portugal; \\ ${ }^{f}$ Li Ka Shing Faculty of Medicine, The University of Hong Kong, Hong Kong Breast Oncology Group (HKBOG), Hong Kong, Hong Kong
}

The International Consensus Conference for Advanced Breast Cancer (ABC) has established itself as a major international breast cancer conference. Its primary aim is the development of international consensus guidelines for the management of $\mathrm{ABC}$ patients. These guidelines are based on the most up-to-date evidence and can be used to guide treatment decision making in many different health care settings globally, with the necessary adaptations due to different access to care. With the motto 'Bridging the Gap', ABC4, which again took place in Lisbon, Portugal in November 2017, brought together over 1,400 participants from 84 countries around the world, including health professionals, patient advocates and journalists. Here, experts from different countries describe their personal highlights of the meeting.

The next ABC meeting will be held in Lisbon in November 2019, see $w w w . a b c$-lisbon.org for details.

Rachel Wuerstlein

\section{Question 1: What Were Your Highlights of this ABC?}

Ettl: There were two definite highlights this year: The best abstract presentation, in my eyes, was given by Nadia Harbeck who presented data from the post-hoc analysis of PALOMA-2 aiming to assess patient reported heath related qualitiy of life (HRQoL) in patients who did progress compared to those who did not. For the first time it could be shown in a prospective, randomized trial in ER+, HER2- advanced/metastatic breast cancer (ABC/MBC), that a delay in progression is associated with a delay of deterioration of
HRQOL. This finding is of great importance in the debate of the question whether progression-free survival (PFS) should be considered a patient relevant endpoint in the absence of overall survival data. The second highlight was the session 'Lost in Translation'. Fabrice Andre, Nick Turner, and George Sledge gave a magnificent overview over the current evidence concerning the use of precision medicine techniques, such as multigene testing and circulating tumor DNA (ctDNA) in MBC. Fabrice Andre presented broad evidence that it indeed makes sense to implement multigene sequencing into clinical trials. Nick Turner stated that although at the moment there is no clinical utility of ctDNA, its analysis can be used for tumor genotyping and should be incorporated into intervention studies to further test them as new markers for guiding systemic therapies.

Haidinger: The award lecture of Musa Mayer was one of my personal highlights. She works in patient advocacy for more than 25 years and shared her experience, pointing out that the public needs to learn that MBC and its impact on the patient, their families and their overall life differs much from early breast cancer. Further, I was impressed by the presentation of Bella Kaufmann, who has been treating breast cancer patients for more than 20 years when she received her own breast cancer diagnosis. Today she has metastatic breast cancer and revealed the daily struggle as oncologist and patient as the same time. Important topics from my point of view were: i) end-of-life communication - patient and health care provider perspectives, e.g. how to communicate that no cancer treatment but treatment for the QoL is an option to be thought of; ii) long-term remissions - challenges in deciding on how and until

\section{KARGER}

() 2017 S. Karger GmbH, Freiburg

Fax +497614520714
Dr. med. Rachel Wuerstlein 
when to treat; iii) the new management of luminal ABC: new treatment options and sequences taking into account the QoL and the importance of a longer time of PFS.

Lueftner: My personal highlight is the strong voice that was raised to use appropriate QoL questionnaires in phase III clinical trials. Specialists complained that while good tools would be available, unthoughtful use of moderate quality questionnaires prevents the acquaintance of highly important clinical data and patient reported outcome results.

Ribeiro: The highlight of this 4th edition of the International Consensus Conference for Advanced Breast Cancer (ABC4) was the outstanding consensus session that took place on the last day Saturday 4th November. It was an amazing opportunity to see the expert's points and discussions regarding evolving issues, such as the benefit of new substances.

Tsang: For this year's ABC4, I would suggest to highlight the recommendation that young women with HR+HER2- ABC should have adequate ovarian suppression or ablation (OS/OA) and then be treated in the same way as post-menopausal women with endocrine therapy and targeted therapy. While previously many of the clinical trials dedicated for HR+HER2- ABC have not included pre-menopausal women, future trials exploring new endocrineeased strategies should be designed to allow for enrolment of both pre- and post-menopausal women. With regard to available options of endocrine therapy, these include aromatase inhibitors (AI), tamoxifen, fulvestrant, AI + cyclin-dependent kinases (CDK), fulvestrant $+\mathrm{CDK}, \mathrm{AI}+$ everolimus, tamoxifen + everolimus, fulvestrant + everolimus.

\section{Question 2: Are the ABC4 Guidelines, Regarding the Consensus of Saturday, in Accordance with Your National Guidelines, or Are There Differences?}

Ettl: This question I find difficult to answer, because the wording of the consensus decisions was very much argued on, thus one should wait for the definite consensus to be published before answering this question.

Lueftner: The guidelines are in line with the national guidelines as they were not agreed upon by availability but purely based on scientific data. However, also these guidelines are not free from individual interpretation. My strongest discord is in terms of the use of checkpoint inhibitors in advanced breast cancer, which is basically not recommended outside of clinical trials. My personal suggestion is that in those patients where the current data suggest a probability of success of $>20 \%$ (triple-negative breast cancer (TNBC), PDL-1 expression $>1$, high LDH levels, maybe lymphocyte infiltration), the use as an individual decision is justified.
Ribeiro: The ABC4 guidelines serve as a major reference for the development of the Portuguese national guidelines.

Tsang: The presence of national guidelines varies among different countries in Asia. Some countries, such as Taiwan, do have their national guideline, but we do not have any national guidelines in Hong Kong. While each institution has its own 'guideline', the Hong Kong Breast Oncology Group (HKBOG) has drafted a local consensus on HR+ HER2- ABC. There are differences in historical or cultural background across Asian countries and being mindful of this helps to engage everyone to be involved in an multidisciplinary team.

\section{Question 3: MBC HER2+ - Do You Expect Improvements in the Near Future?}

Ettl: With the introduction of biosimilars I expect the 'financial toxicity' to go down. As a consequence, hopefully resources will be set free to be used for the development of other new effective drugs.

Lueftner: Marginal improvements are possible if modern tyrosine kinase inhibitors (TKI) like tucatinib are successful and help to treat or even postpone brain metastases. However, this is a hypothesis and must be proven in larger phase III clinical trials.

Ribeiro: The major improvements that I expect soon regarding HER2 + ABC are related to the development of agents to treat brain metastases. Patients with HER $2+$ ABC have a $50 \%$ chance of developing brain metastases so this is a huge problem and an unmet need. Agents like tucatinib and other TKI have shown promising results and further studies are eagerly awaited.

Tsang: Yes, especially with emerging new anti-HER2 therapy options over the recent years, there should be further personalized medicine available to be delivered to our patients. Of course, access hurdles and matching science with affordability is always a challenge in Asia.

\section{Question 4: Triple-Negative MBC: How Will Personalized / Molecular-Oriented Medicine Change Management of this Subtype and How Fast?}

Ettl: In my opinion it is too early and data are not sufficient to sound into the hype of targeting new molecular mechanisms in TNBC.

Lueftner: ABC4 underlined the fact that standardized detection of the androgen receptor is missing, and based on that, the use on bicalutamid or enzalutamide must be considered investigational. This is even more important as it seems that there will not be any phase III trials in the near future. 
Ribeiro: Personalized/molecular-oriented medicine is a fastgrowing field that poses many practical, legal, and ethical challenges. At this moment from my point of view the main application of a molecular-oriented management in $\mathrm{TN} \mathrm{ABC}$ is related to the use of PARP inhibitors in patients with BRCA germline mutations. I believe that the use of microsatellite instability (MSI-H) or mismatch repair deficient (dMMR) biomarkers to drive immunotherapy will be the next important achievement.

Tsang: Personalized / molecular-oriented medicine will further identify intrinsic subtypes of the TNBC, and this may further help to best select subtypes predicted to be responding well towards new agents, e.g. in immuno-oncology. This molecular driven approach will lead us to precision medicine in a fast manner.

\section{Question 5: ABC Luminal Type: What Is the Role of CDK4/6 Inhibitors at Your Clinics?}

Ettl: In my clinic combination of endocrine therapy with CDK4/6 inhibitor is the new standard of care for treating patients with HR+, HER2- MBC.

Lueftner: Knowing that there is no predictive marker indicating any special advantage from the use of a CDK4/6 inhibitor, and due to the clinically important prolongation of PFS, CDK4/6 inhibitors are a standard of care in our clinical routine while endocrine monotherapy starts to become an exception.

Ribeiro: The use of CDK4/6 inhibitors is a regular practice in my clinic nowadays with the main application being in combination with an AI as initial therapy or in combination with fulvestrant in women who have received prior endocrine therapy.

Tsang: There is indeed a role and added value of CDK4/6 inhibitors in our clinics back in Asia, based on the emerging data from various clinical trials, and there is more than one type of CDK4/6 inhibitors available these days. There is a role of CDK4/6 inhibitors as first-line treatment for HR+ HER2- ABC and when a patient is anticipated to be a 'fast progressor'. There is also a role for CDK4/6 inhibitors beyond the 1st line of treatment.

\section{Question 6: Will Local Treatment Options Change Our Approach in MBC?}

$E t t l$ : Local treatment options, such as surgery, radiation therapy and also electrochemotherapy will always have to be integrated into systemic therapies for $\mathrm{MBC}$ at differnet timepoints in the course of the disease.

Lueftner: No. Currently, there are no safe data supporting the generalized use of local treatment options in MBC. Unless there is oligometastatic disease which is controlled for a long time, one might consider local treatment options after thorough information about the advantages and disadvantages. In any case, if parallel systemic treatment is possible, it must not be withheld from the patient.

Ribeiro: Local treatment options are an important part of the therapeutic armamentarium of $\mathrm{ABC}$. In this era of targeted therapy, where we see major and longstanding responses, the application of local treatment options will be increasingly recognized. For now, however, $\mathrm{ABC} 4$ recommendations regarding surgery didn't change compared to ABC3.

Tsang: Loco-regional treatment with radiation or stereotactic body radiotherapy might be used for better control of disease, also debulking surgery for removal of the primary tumor for patients presenting with de novo disease. This is especially true for patients presenting with oligometastases.

\section{Question 7: New Substances: How Can We Better Manage the Gap between Development of New Drugs and Early Clinical Availability?}

Ettl: The process of drug approval and introduction into the national markets should be parallelized. The pharma industry should get the chance to disclose the costs of drug development and explain their pricing strategies.

Lueftner: The scientific community must take special care with 'orphan diseases' within breast cancer. We need to make better use of computer techniques and international registries to detect rare, targetable lesions earlier.

Ribeiro: I do believe that the deeper and better knowledge of breast cancer biology will enable us to develop different study designs focusing on restricted patient populations and leading to faster drug approvals.

Tsang: At the moment, the general availability of the new drugs is very uneven around the world. To better manage the gap, we should help to facilitate raising the awareness among patients and their carers of these new agents. Cost of the drug and lack of full reimbursement of any new agents is always a challenge. Fund raising to support the underprivileged to have equal access is a good direction to go.

\section{Question 8: Where Do We Need Improvement for Better Psychosocial Care of Patients in the Metastatic Setting and How Can this Be Realized?}

Ettl: We do need earlier integration of palliative care into the management of ABC Patients. Palliative care givers should be aware that anti-tumor drugs are 'symptom oriented therapies' in a 
similar way as palliative care is. These different treatments should not be weighed against each other.

Haidinger: Awareness for ABC, especially metastatic breast cancer, has to be improved for a better understanding of the patients' burden of the disease, including its economic burden. We should gather 'real-world data', seek patient input on the value assessment of cancer treatment and care, and the wide differences in access of treatment and care among the different countries. More effort must be put into giving patients easy access to psychooncologic support.

Lueftner: As we have learnt from $\mathrm{ABC} 4$, empathy reduces the necessary therapeutic distance to the patient with the danger of unrealistic information of the patient about the potential benefit from systemic therapies. We must keep on investigating this psychological feature to bring the relationship between physician and patient onto a level that remains honest throughout the long course of the disease.

Ribeiro: As physicians, we need to acknowledge the importance of early referral of these patients and families to psychooncological care and actively support research in this setting.

Tsang: We should address the unmet psychosocial needs of patients living with MBC. A holistic approach for management of MBC patients should be advised and encouraged. Patients' wishes concerning quality of life, understanding of toxicity profile, and preference of treatment, such as oral vs. i.v. systemic chemotherapy should be taken into account. Dose reductions may sometimes be valid, especially when this helps to avoid interruption of any drug. Survivorship should be advocated and supported.

\section{Question 9: Patients Advocacy Plays a Major Role at $A B C$ and Its Consensus - Where Do You See Possible Improvements for Patients Advocacy in the Metastatic Setting in Your Surrounding?}

Ettl: Patients' advocates could be a big help in communicating early with $\mathrm{ABC}$ patients about palliative and end of life care.

Haidinger: Patients' advocates can teach health authorities about the vast difference between metastatic disease and early breast cancer and they could support health technology assessment methodologies to be adapted to the reality of $\mathrm{MBC}$ care and treatment. They can improve the understanding of physicians of the value patients place on a considerably longer progression-free survival with acceptable QoL, even though the main goal always has to be prolonged overall survival.

Lueftner: Patient advocates are an important bridge between patients and physicians. At ABC4, we heard presentations from advocates who are affected of MBC themselves, and those who are not.
It seems that this mixture is helpful not to dive too deep into psychosocial aspects and keep objective distance. This is necessary to retain an effective relationship to political opinion leaders and health technology assessment institutions.

Ribeiro: A focus of the advocacy discussion at this meeting related to employment issues faced by patients with $\mathrm{ABC}$. It was very interesting to see the diversity of patient's preferences and realize the need for political changes in this field that allow individualized options. We need an update of employment legislation and adjustments to support a return to work.

Tsang: Asian patients and carers tend to be more reserved. But with the inspiration and encouragement from Musa Mayer who is a patient advocate, and awardee of this year's $\mathrm{ABC}$ award, this will empower many more of our Asian patients to voice out their concerns and unmet needs. Patient advocacy plays an important role in empowering patients and carers.

\section{Question 10: Was there Practice-Changing Information You Will Take into Account for Your Clinical Management Right after ABC?}

Haidinger: We should talk about palliative care and its advantages right after diagnosis of metastatic disease and produce a manual for patients to guide them. It should inform them about all the support opportunities offered for them and about their rights.

Lueftner: The stress that was put on MSI-H positivity and NTRK mutations will induce more testing for the rare event in my clinical practice.

Ribeiro: The ABC4 took place in a great science-driven discussion environment. Several important and controversial topics concerning the management of $\mathrm{ABC}$ where deeply reviewed and discussed among the expert panel, audience, and patient advocates. Some hot topics where CDK4/6 inhibitors, biosimilars or olaparib use. The incorporation of molecular assays and liquid biopsies technology, although not readily available to use in daily practice, was discussed and will be one of the great innovations of oncological practice in the near future.

Tsang: We should treat both pre-menopausal and post-menopausal HR+ HER2- ABC patients the same, provided that the young women have prior adequate ovarian suppression or ablation, and future study designs should be able to embrace and include both pre-menopausal and post-menopausal women to be able to participate in these studies. 


\section{Participants}

Dr. Johannes Ettl

Frauenklinik, Klinikum rechts der Isar

Technical University Munich

Ismaninger Str. 22, 81675 Munich, Germany

johannes.ettl@tum.de

\section{Ms. Renate Haidinger}

Brustkrebs Deutschland e.V.

Lise-Meitner-Str. 7, 85662 Hohenbrunn, Germany

renate.haidinger@brustkrebsdeutschland.de

\section{Prof. Dr. Diana Lueftner}

Medical Clinic focusing on Hematology, Oncology and Tumor Immunology Charité Berlin, Benjamin Franklin Campus

Hindenburgdamm 30, 12200 Berlin, Germany

diana.lueftner@charite.de
Dr. Joana Ribeiro

Champalimaud Clinical Centre

Avenida Brasília, 1400-038 Lisboa, Portugal

joana.ribeiro@fundacaochampalimaud.pt

Dr. Janice Tsang

Li Ka Shing Faculty of Medicine

The University of Hong Kong

Hong Kong Breast Oncology Group (HKBOG)

Room 825, 8/F Tower A (Pacific Link Tower), Southmark,

11 Yip Hing Street, Wong Chuk Hang, Hong Kong, Hong Kong SAR janicetsangmedon@gmail.com 